\title{
BMJ Open The utility of Ki-67 as a prognostic biomarker in pulmonary neuroendocrine tumours: protocol for a systematic review and meta-analysis
}

\author{
Salma Naheed, ${ }^{1}$ Chloe Holden, ${ }^{2}$ Lulu Tanno, ${ }^{1}$ Eleanor Jaynes, ${ }^{3}$ Judith Cave, ${ }^{2}$ \\ Christian H Ottensmeier, ${ }^{1}$ Giuseppe Pelosi ${ }^{4}$
}

To cite: Naheed S, Holden C, Tanno L, et al. The utility of $\mathrm{Ki}-67$ as a prognostic biomarker in pulmonary neuroendocrine tumours: protocol for a systematic review and meta-analysis. BMJ Open 2019;9:e031531. doi:10.1136/ bmjopen-2019-031531

- Prepublication history and additional material for this paper are available online. To view these files, please visit the journal online (http://dx.doi. org/10.1136/bmjopen-2019031531).

Received 08 May 2019 Revised 28 June 2019 Accepted 12 July 2019
Check for updates

(C) Author(s) (or their employer(s)) 2019. Re-use permitted under CC BY-NC. No commercial re-use. See rights and permissions. Published by BMJ.

For numbered affiliations see end of article.

Correspondence to

Dr Salma Naheed;

s.naheed@soton.ac.uk

\section{ABSTRACT}

Introduction The omission of the immunohistochemical proliferation marker Ki-67 labelling index (henceforth, simply Ki-67) from the 2015 WHO classification system of pulmonary neuroendocrine tumours (Lung-NETs) as a prognostic and grading criterion remains controversial. This systematic review along with meta-analysis will be conducted to assess the prognostic/grading utility of Ki-67 in Lung-NETs.

Methods This systematic review will be conducted in accordance with the Preferred Reporting Items for Systematic Reviews and Meta-Analysis guidelines. A systematic search of MEDLINE Ovid, Embase, Scopus and the Cochrane Library will be performed from the inception of each database to 28 February 2019 for studies investigating any role of Ki-67 in Lung-NETs. Only full papers published in English detailing survival outcomes and HRs according to Ki-67 will be included. The primary endpoint will be establishing whether Ki-67 is a reliable marker in determining prognosis and thus assessing grade of Lung-NETs patients.

Ethics and dissemination Ethical approval will not be required as this is an academic review of published literature. Findings will be disseminated through the preparation of a manuscript for publication in a peerreviewed journal as well as presentation at national and international conferences.

PROSPERO registration number CRD42018093389

\section{INTRODUCTION}

Neuroendocrine tumours (NETs) represent an extremely heterogenous group of tumours. Almost any anatomical site can be affected and the clinical behaviour of these tumours also varies considerably. Approximately $27 \%$ of all NETs arise within the lungs and the incidence of pulmonary neuroendocrine tumours (LungNETs) is increasing for reasons which are still unclear. ${ }^{1-3}$ One factor is likely to be an increase in incidental diagnoses as a result of advances in diagnostic imaging performed for unrelated reasons.

$\mathrm{Ki}-67$ is a DNA-binding nuclear protein which was discovered by Gerdes et al in $1983 .{ }^{4}$

\section{Strengths and limitations of this study}

- A comprehensive review of the utility of Ki-67 as a prognostic marker in pulmonary neuroendocrine tumours will be performed following the key methodological steps of Preferred Reporting Items for Systematic Review and Meta-Analysis Protocols guidelines.

- A meticulous search will be carried out for the relevant studies from several databases endeavouring to capture all the relevant published literature.

- Papers not published in English will be excluded and therefore, significant outcomes could be overlooked.

- It is anticipated that there may be significant interstudy heterogeneity in how results will be reported, the cut-off points for Ki-67 percentage or other issues. Accordingly, it may not be possible to conduct a meta-analysis, particularly if only a limited number of articles are identified with sufficient information.

It is expressed throughout all phases of the cell cycle, besides the quiescent G0 phase. As a result, its main clinical utility is in distinguishing between proliferating and non-proliferating cells. $\mathrm{Ki}-67$ is a robust biomarker of proliferation in malignancy given its qualities of tight cell cycle regulation and rapid degradation after the completion of mitosis (Ki-67 has a short half-life of $1-1.5$ hours), where it acts as a biological surfactant to disperse mitotic chromosomes. ${ }^{5}$ The gene encoding the Ki-67 protein has been termed MKI67 and is located on chromosome $10 \mathrm{q} 26 .{ }^{6}$

Most frequently, Ki-67 is quantified by immunohistochemistry as a score or labelling index (LI) based on the percentage of tumour cells which stain positively (usually a minimum of 500 cells, with best practice being to count 2000 cells or $2 \mathrm{~mm}^{2}$ ) usually in areas of most dense staining. ${ }^{78} \mathrm{Ki}-67$ is regarded as a more accurate measure of cell proliferation in comparison to mitotic figure counting. Immunohistochemical evaluation, however, is not without its 
challenges. Manual tumour cell counting is onerous, typically taking $\sim 40 \mathrm{~min}$ to complete when using slide photography and/or other digital methods to prepare a field for tumour cell counting of the required number of cells. Some pathologists therefore use the 'eyeball' counting method which is less accurate, and varies according to pathologist's experience. This subjective approach is closely related to the observers' experience and thus has been criticised for its imprecision resulting in interobserver variability ${ }^{9}$ Another proposed method to achieve uniformity and improve the practicality of counting (in terms of time limitations) is digital image analysis. However, computer-assisted methods are not currently in widespread use due to lack of availability and technical inaccuracy in overcounting unwanted cells or underestimating negative elements. Low interobserver variation has been heralded by using camera-captured printed images, but the problem still remains in the choice of tumour areas subjected to counting due to the huge intra-tumoural heterogeneity of $\mathrm{Ki}-67$, thus leading to significantly different scores. ${ }^{10}$ Further complexity is that Ki-67 may change over time either due to the natural history of the disease or as a result of treatment-based pressures. ${ }^{11}$

Multiple studies across a range of malignancies (eg, breast, prostate and NETs at several anatomical sites) have determined that there is a significant association between the tumour proliferation as assessed by $\mathrm{Ki}-67 \mathrm{LI}$ and the patient survival. ${ }^{16-16}$ In breast cancer, two significant meta-analyses dealing with over 40000 patients demonstrated the independent prognostic value of Ki-67, with high Ki-67 indices being associated with incremental risk of relapse together with poorer overall survival in both node negative and node positive disease. ${ }^{12}{ }^{17}$ Nevertheless, the debate regarding the intrinsic reliability issues continues among clinicians, with the American Society of Clinical Oncology tumour marker expert panel on breast cancer stating that it does not recommend the use of Ki-67 to assess prognosis. ${ }^{18}$ However, further research into the utility of Ki-67 as a prognostic biomarker is warranted given its advantages compared with newer genomic based biomarkers, including low cost, worldwide experience of the use of this marker by pathologists, clinical relevance and reproducible results when strict counting guidelines are applied. ${ }^{19}$

Lung-NETs are classified by the 2015 WHO on morphology grounds, ranging from well-differentiated low-grade typical carcinoids and intermediate-grade atypical carcinoids to poorly differentiated high-grade large cell neuroendocrine carcinoma and small cell lung carcinoma (SCLC) with no significant differences in survival between them. ${ }^{20}$ Therefore, while mitotic activity and necrosis are key factors within this classification system, these tumours are not further stratified into grades (table 1). Within the WHO 2015 classification, the only permissible role for Ki-67 is limited to the diagnostic separation of carcinoids from SCLC, mainly in the setting of biopsy or cytological samples with crush artefacts. This places pulmonary NETs at odds with gastroenteropancreatic (GEP) NETs where Ki-67 was proposed for the first time for predicting survival. Subsequently, it has been used together mitotic count for tumour grading with a predominant role of Ki-67 over mitoses for dictating ultimate clinical outcomes (table 2). ${ }^{21-23}$ As a result, in GEP-NETs, Ki-67 has become highly influential in determining management approaches. More recently, the updated 2017 WHO classification for pancreatic neuroendocrine neoplasms included a formal recognition of the heterogeneity of pancreatic NETs. ${ }^{24}$ The amended criteria now contains not only a poorly differentiated grade 3 neuroendocrine carcinoma group but also a distinct well-differentiated grade 3 NET group. However, this distinction has not been extended more widely despite a recent grading proposal of resected Lung-NETs (assessing Ki-67, mitotic count and necrosis) which suggests that this would be a valid distinction. ${ }^{25}$

The omission of Ki-67 in the Lung-NET cohort is likely to be due to a perceived lack of an evidence base surrounding its role as an independent prognostic factor. ${ }^{811}$ Nonetheless, Ki-67 LI is often required by oncologists for therapy planning and prognostic/grading assessment of these tumours. ${ }^{26}$ The aim of this systematic review and meta-analysis is to determine whether existing studies support Ki-67 as a prognostic (and by extension grading) biomarker in the management of pulmonary neuroendocrine neoplasms.

\section{METHODS AND ANALYSIS}

In accordance with Preferred Reporting Items for Systematic Reviews and Meta-Analyses (PRISMA) guidelines, a prospective protocol has been produced and registered in the PROSPERO International Registry. ${ }^{27}$

Table 1 Modified from WHO diagnostic criteria for pulmonary NETs 2015

\begin{tabular}{lllll} 
& TC & AC & LCNEC & SCLC \\
\hline Mitoses & $<2$ & $2-10$ & $>10$ & $>10$ \\
Necrosis & Absent & $\begin{array}{l}\text { If present, usually focal or } \\
\text { punctate }\end{array}$ & Extensive & Extensive \\
Morphology & Organoid & Organoid & Organoid & Small cell \\
Differentiation grade & Low & Intermediate & High & High
\end{tabular}

AC, atypical carcinoid; LCNEC, large cell neuroendocrine carcinoma; NETs, neuroendocrine tumours; SCLC, small cell lung carcinoma; TC, typical carcinoid. 


\begin{tabular}{ll} 
Table 2 & WHO 2017 grading system for GEP-NETs \\
\hline Grade & Definition \\
\hline 1 & Mitotic count $<2 / 10$ HPF; Ki-67 $<3 \%$ \\
2 & Mitotic count $=2-20 / 10$ HPF OR Ki-67 $=3 \%-20 \%$ \\
3 & Mitotic count $=>20 / 10$ HPF OR Ki-67 $>20 \%$ \\
\hline
\end{tabular}

Bold Values denotes that only one of the two criteria are needed for classification.

GEP, gastroenteropancreatic; NETs, neuroendocrine tumours.

\section{Selection criteria}

For inclusion in this review, studies (both retrospective and prospective) will be required to provide survival data for a cohort of patients with Lung-NETs. Only studies available in full text reporting original data in English will be included in the review. Key exclusion criteria include studies published after 28 February 2019, as well as studies only available in abstract form. (For full eligibility criteria, see table 3.)

\section{Search strategy}

To identify relevant studies, a search will be conducted utilising the following electronic databases: Medline Ovid, EMBASE, the Cochrane Library and Scopus. The search strategy developed in Medline Ovid uses a combination of Mesh subject headings and free text terms around the two key components of ' $\mathrm{Ki}-67$ ' and 'neuroendocrine tumours'. An example of the full search strategy is available in online supplementary file 1 . This search strategy was then modified for use in the other databases. Furthermore, reference lists of included studies and relevant reviews will be screened for any relevant primary studies which were not identified through the database search.

\section{Study selection}

Rayyan software will be employed to facilitate the initial screening process and duplicated records will be removed. Two independent reviewers ( $\mathrm{SN}$ and $\mathrm{CH}$ ) will screen the titles and abstracts identified through the different databases using the prespecified eligibility criteria. This will be completed in a blinded fashion. The primary reason for elimination will be noted for all exclusions. The results will be cross-referenced, prior to analysis. In case of disagreements, a third reviewer (GP) will adjudicate and then a consensus decision will be reached. Those studies deemed to meet the initial screening will then undergo a further full text screening against the full inclusion criteria prior to inclusion in the systematic review. The study selection process will be reported graphically using the PRISMA flow diagram.

\section{Data extraction}

Data will be extracted by one author (SN) under the supervision of a second author (GP) using an agreed, standard data extraction spreadsheet in Microsoft Excel. A random selection of five papers will be used to pilot the data extraction spreadsheet and modifications will be made if necessary. The following data will be collected (see box 1 ):

1. Study design characteristics: for example, prospective or retrospective, methodology for calculating Ki-67, length of follow-up, statistical methods.

2. Population characteristics: for example, histological subtypes, patient demographics, staging.

3. Outcomes: for example, 5-year survival statistics, HRs (and CIs).

\section{Assessment of study quality}

To facilitate evaluation of the quality of studies eligible for the meta-analysis, the Newcastle-Ottawa scale will be utilised. ${ }^{28}$ This tool will be used to assess each individual study against the following criteria: case selection, flow of patients through the study, clear methodology for Ki-67 index calculations, as well as outcome measurement.

\section{Table 3 Eligibility criteria}

\section{Inclusion criteria}

Any studies of Ki-67 in pulmonary neuroendocrine neoplasms providing prognostic information.

\section{Exclusion criteria}

Non-human studies.

Non-pulmonary neuroendocrine tumours.

Combined histological subtypes that is, both combined neuroendocrine neoplasms or combined non-small cell lung carcinomaswith lung NETs.

Studies not available as a full manuscript.

Less than 20 cases.

Studies published after 28 February 2019.

Review papers and case reports.

Studies not published in English.

NETs, neuroendocrine tumours. 


\section{Box 1 Data items}

\section{Study characteristics}

- Author.

- Year of publication.

- Study design.

- Antibody utilised.

Methodology for calculating Ki-67.

- Length of follow-up.

Statistical method.

For each histological subtype (ie, typical carcinoid, atypical carcinoid, large cell neuroendocrine cancer, small cell lung cancer)

- Patient number.

- Mean age at diagnosis (range).

- Male:female ratio.

- Tumour, node, metastases stage at diagnosis.

- Any prior treatment received.

- Tumour diameter (cm).

- Lymph node metastases at diagnosis (ratio of present:absent:unknown).

- Distant metastases at diagnosis (ratio of present:absent:unknown).

Ki67 cut-off point (\%).

Outcomes

- Overall survival.

- Disease-free survival.

- HRs (and Cls).

Results (including p values).

\section{Assessing risk of bias}

The risk of publication bias will be evaluated using funnel-plot visual inspection in conjunction with Begg's and Egger's test.

\section{Data synthesis and analysis}

A descriptive analysis together with summary tables of the characteristics of studies included in the systematic review will be completed. In order to determine whether a meta-analysis is feasible, an analysis of study heterogeneity will be undertaken. It is anticipated that the main source of study heterogeneity will be in the form of Ki-67 cut-offs utilised as well as the clinical end-points used to define outcomes. Given the likelihood of variability between included studies, a random-effects meta-analysis will be used to calculate the combined estimates of survival proportions.

\section{Patient and public involvement}

Patients and the public were not involved in the development of this systematic review protocol.

\section{ETHICS AND DISSEMINATION}

Given that this is a systematic review of existing literature, ethical approval is not required. Findings from the systematic review and meta-analysis will be submitted for publication in a peer-reviewed journal.
Author affiliations

${ }^{1}$ Cancer Sciences Unit, NIHR and CRUK Experimental Cancer Medicine Center and NIHR Biomedical Research Center Southampton, University of Southampton Faculty of Medicine, Southampton, UK

${ }^{2}$ Department of Oncology, Wessex NET Group ENETS Centre of Excellence, University Hospital Southampton NHS Foundation Trust, Southampton, UK ${ }^{3}$ Department of Cellular Pathology, Wessex NET Group ENETS Centre of Excellence, University Hospital Southampton NHS Foundation Trust, Southampton, UK

${ }^{4}$ Department of Oncology and Hemato-Oncology, University of Milan, Milano, Italy

Contributors SN contributed to the study question, protocol design and will be involved in screening, data collection, data analysis and dissemination of results. $\mathrm{CH}, \mathrm{LT}, \mathrm{JC}$ and EJ have contributed to the protocol design. CHO and GP were responsible for the study question; all authors have been involved in writing this protocol. They will also be involved in the preparation of any manuscripts arising from this review.

Funding SN's postgraduate studies are funded by PLANETS Cancer Charity, a not for profit organisation which had no role in developing this protocol.

Competing interests None declared.

Patient consent for publication Not required.

Provenance and peer review Not commissioned; externally peer reviewed.

Open access This is an open access article distributed in accordance with the Creative Commons Attribution Non Commercial (CC BY-NC 4.0) license, which permits others to distribute, remix, adapt, build upon this work non-commercially, and license their derivative works on different terms, provided the original work is properly cited, appropriate credit is given, any changes made indicated, and the use is non-commercial. See: http://creativecommons.org/licenses/by-nc/4.0/.

\section{REFERENCES}

1. Yao JC, Hassan M, Phan A, et al. One Hundred Years After "Carcinoid": Epidemiology of and Prognostic Factors for Neuroendocrine Tumors in 35,825 Cases in the United States. JCO 2008;26:3063-72.

2. Modlin IM, Lye KD, Kidd M. A 5-decade analysis of 13,715 carcinoid tumors. Cancer 2003;97:934-59.

3. Travis WD, Travis LB, Devesa SS. Lung cancer. Cancer 1995;75(1 Suppl):191-202.

4. Gerdes J, Schwab U, Lemke H, et al. Production of a mouse monoclonal antibody reactive with a human nuclear antigen associated with cell proliferation. Int J Cancer 1983;31:13-20.

5. Cuylen S, Blaukopf C, Politi AZ, et al. Ki-67 acts as a biological surfactant to disperse mitotic chromosomes. Nature 2016;535:308-12.

6. Menon SS, Guruvayoorappan C, Sakthivel KM, et al. Ki-67 protein as a tumour proliferation marker. Clinica Chimica Acta 2019;491:39-45.

7. Fabbri A, Cossa M, Sonzogni A, et al. Ki-67 labeling index of neuroendocrine tumors of the lung has a high level of correspondence between biopsy samples and surgical specimens when strict counting guidelines are applied. Virchows Arch 2017;470:153-64.

8. Pelosi G, Rindi G, Travis WD, et al. Ki-67 antigen in lung neuroendocrine tumors: unraveling a role in clinical practice. $J$ Thorac Oncol 2014;9:273-84.

9. Klimstra DS, Modlin IR, Adsay NV, et al. Pathology reporting of neuroendocrine tumors: application of the Delphic consensus process to the development of a minimum pathology data set. Am J Surg Pathol 2010;34:300-13.

10. Reid MD, Bagci P, Ohike N, et al. Calculation of the Ki67 index in pancreatic neuroendocrine tumors: a comparative analysis of four counting methodologies. Mod Pathol 2015;28:686-94.

11. Pelosi G, Pattini L, Morana G, et al. Grading lung neuroendocrine tumors: controversies in search of a solution. Histol Histopathol 2017;32:223-41.

12. de Azambuja E, Cardoso F, de Castro G, et al. Ki-67 as prognostic marker in early breast cancer: a meta-analysis of published studies involving 12,155 patients. Br J Cancer 2007;96:1504-13.

13. Khor L-Y, Bae K, Paulus R, et al. Mdm2 and Ki-67 predict for distant metastasis and mortality in men treated with radiotherapy and androgen deprivation for prostate cancer: RTOG 92-02. JCO 2009;27:3177-84.

14. Jann H, Roll S, Couvelard A, et al. Neuroendocrine tumors of midgut and hindgut origin: tumor-node-metastasis classification determines clinical outcome. Cancer 2011;117:3332-41. 
15. Klöppel G, La Rosa S. Ki67 labeling index: assessment and prognostic role in gastroenteropancreatic neuroendocrine neoplasms. Virchows Arch 2018;472:341-9.

16. Richards-Taylor S, Ewings SM, Jaynes E, et al. The assessment of $\mathrm{Ki}-67$ as a prognostic marker in neuroendocrine tumours: a systematic review and meta-analysis. J Clin Pathol 2016;69:612-8.

17. Stuart-Harris R, Caldas C, Pinder SE, et al. Proliferation markers and survival in early breast cancer: a systematic review and metaanalysis of 85 studies in 32,825 patients. Breast 2008;17:323-34.

18. Harris L, Fritsche H, Mennel R, et al. American Society of clinical oncology 2007 update of recommendations for the use of tumor markers in breast cancer. JCO 2007;25:5287-312

19. Young HTM, Carr NJ, Green B, et al. Accuracy of visual assessments of proliferation indices in gastroenteropancreatic neuroendocrine tumours. J Clin Pathol 2013;66:700-4.

20. Travis WD, Burke AP, Marx A, et al. Who Classification of tumours of the lung, pleura, thymus and heart. Int Agency Res Cancer 2015.

21. Bosman F, Carneiro F, Hruban R, et al. E. who classification of tumours of the digestive system. Lyon, France: IARC Press, 2010.

22. Lloyd RV, Osamura RY, Klöppel GRJ. Who classification of tumours of endocrine organs. 4th edn, 2018.
23. Pelosi G, Bresaola E, Bogina G, et al. Endocrine tumors of the pancreas: Ki-67 immunoreactivity on paraffin sections is an independent predictor for malignancy: a comparative study with proliferating-cell nuclear antigen and progesterone receptor protein immunostaining, mitotic index, and other clinicopathologic variables. Hum Pathol 1996;27:1124-34.

24. Inzani F, Petrone G, Rindi G. The new World Health organization classification for pancreatic neuroendocrine neoplasia. Endocrinol Metab Clin North Am 2018;47:463-70.

25. Rindi G, Klersy C, Inzani F, et al. Grading the neuroendocrine tumors of the lung: an evidence-based proposal. Endocr Relat Cancer 2014;21:1-16.

26. Marchevsky AM, Hendifar A, Walts AE. The use of Ki-67 labeling index to grade pulmonary well-differentiated neuroendocrine neoplasms: current best evidence. Mod Pathol 2018;31:1523-31.

27. Moher D, Liberati A, Tetzlaff J, et al. Preferred reporting items for systematic reviews and meta-analyses: the PRISMA statement. PLoS Med 2009;6:e1000097.

28. McShane LM, Altman DG, Sauerbrei W, et al. Reporting recommendations for tumour marker prognostic studies (REMARK). Br J Cancer 2005;93:391. 